Category: Teaching \& Learning at times of uncertainty

\title{
Usefulness of AMBOSS enhanced e-learning in COVID situation: feedback from medical students
}

\author{
Mitra, N.K., Kumar, A. ${ }^{1}$, Baloch, H.Z. ${ }^{1}$, Nadarajah, V.D. ${ }^{1}$, Höller-Fam ${ }^{2}$, M., Schmok, E. ${ }^{2}$ \\ ${ }^{1}$ International Medical University Malaysia \& ${ }^{2}$ AMBOSS GmbH, Germany
}

\section{Background:}

During the COVID situation, both teachers and students need online integrated multi-mediabased resources. In view of internationalization of medical education, resources developing global outlook are preferred. IMU has tied up with AMBOSS to provide an enhanced learning resource for medical students. Resources like AMBOSS prepare students for standardized examinations like USMLE with global outlook of the medical curriculum. The study's objective was to evaluate the usefulness of the AMBOSS resources and compare its usage between preclinical and clinical students.

\section{Methods:}

A twenty-item questionnaire was constructed based on previous students' feedback on IMU external resources. The input was anonymous and taken with the students' consent. AMBOSS data showed that 1,438 students at IMU ( $91.6 \%$ of eligible students) had used AMBOSS at least monthly. Of these, 150 students responded to the questionnaire. Descriptive statistics of the data received from the feedback was computed and analysed and where possible compared with AMBOSS license statistics.

\section{Results:}

Awareness about this learning resource was higher among pre-clinical students (98.6\%) compared to clinical students (93.6\%). More than $98 \%$ of students agreed that this resource is useful for learning in the medical curriculum. More than $80 \%$ of students agreed that the resource is better than other learning resources and $98 \%$ agreed that it was a good decision by IMU to bring in AMBOSS resources for all students. Equal percentage of students in pre-clinical and clinical phases accessed the resources at least once a week (41-42\%). Clinical students accessed twice a week (58.9\%) compared to pre-clinical students (52\%). A gap was observed in the usage of the AMBOSS mobile application for iOS or Android. Pre-clinical students used the mobile application regularly (60\%), with $14 \%$ not knowing about it. It was apparent that there are differences between the question design used in local assessments versus those found in the AMBOSS QBank. Looking at learning behaviour, more than $92 \%$ of students used the extensive library, while $67 \%$ of pre-clinical students and $71 \%$ of clinical students regularly used the QBank. This is also reflected in the total number of articles read $(438,100)$ vs. the total number of questions completed $(154,236)$. Pre-clinical students expressed that the number of videos was not sufficient, and they expected videos on more topics. Nearly $60 \%$ of pre-clinical students agreed that chalkboard videos were useful. Importantly, $68 \%$ of pre-clinical and $60 \%$ of clinical students agreed that content presented in AMBOSS was not different from what they learn in their syllabus. $16 \%$ to $23 \%$ of students felt that they needed a more focused briefing on the substantial functionalities of the AMBOSS resource. 


\section{Conclusion:}

AMBOSS as an enhanced learning resource that has been very valuable for the students in IMU medical program. Feedback information sent to the AMBOSS team will further strengthen this online resource. Overall, the learning cards have seen higher adoption compared to the QBank. Stronger involvement of faculty in integrating the resource into teaching and the use of the QBank for formative assessments is required,

Keywords: Enhanced Learning Resources. 